\title{
La discrépance isotrope et l'intégration numérique.
}

\author{
S. K. Zaremba (Montréal, Canada) $(*)$
}

Sommaire. - On sait que quand il s'agit d'intégrer une fonction suffisamment régulière sur un pavé de mesure 1 à n'importe quel nombre fini de dimensions en prenant comme la valeur de l'intégrale la moyenne des valeurs de cette fonction sur un ensemble fini de points du pave, des majorantes de l'erieur d'intégration peuvent étre exprimées en fonctions de certains paramètres de l'intégrande et de la discrépance de l'ensemble de points. La note suivante est consacré à une extension de ces résultats au cas de l'intégration d'une fonction sur des domaines convexes arbitraires contenus dans un pavé semblable. Etant donné un ensemble $X$ de $N$ points au pavé, on peut regarder comme une approximation de l'intégrale le produit de $N$ ' par la somme des valeurs de l'in. tégrande aux pints de l'ensemble $X$ qui appartiennent au domaine d'intégration. Une majorante de l'erreur s'exprime alors en fonction des mêmes paramètres de l'intégrande que précédemment, de sa caleur à un point particulier et d'un paramètre de $X$ que l'auteur propose d'appeler la discrépance isotrope de cet ensemble. On obtient aussi une minorante absolue de la discrépance isotrope en fonction du nombre de points_de $l$ ensemble et du nombre de dimensions.

\section{1. - Introduction.}

On sait que l'intégrale d'une fonction $f$ sur un domaine de mesure 1 dans un espace à un nombre arbritraire fini de dimensions peut être approchée par la moyenne de l'intégrande sur un ensemble fini $\boldsymbol{X}=\left\langle\boldsymbol{x}^{(0)}, \ldots, \boldsymbol{x}^{(N-1)}\right\rangle$ de points de ce domaine a condition que $f$ satisfasse certaines conditions de régularité et que l'ensemble $\boldsymbol{X}$ de points ait certaines propriétés d'équidis. tribution. Le cas où le domaine d'intégration est un pavé

$$
0 \leq x_{i}<1 \quad(i=1, \ldots, s)
$$

à $s$ dimensions $(s=1,2, \ldots)$ est assez bien connu ([3], [5], [7]).

Pour la valeur absolue

$$
\left|\frac{1}{N} \sum_{k=0}^{N-1} f\left(\boldsymbol{x}^{(k)}\right)-\int_{Q^{s}} f(\boldsymbol{x}) d \boldsymbol{x}\right|
$$

de l'errear d'intégration on obtient des majorantes favorables quand $f$ est à variation bornée au sens de $H_{A R D X}$ et $\mathrm{K}_{\mathrm{RAUSE}}$ sur $Q^{s}$ et la discrépance de $\boldsymbol{X}$

(*) Entrata in Redazione il 28 febbraio 1970. 
est petite; on va rappeler un pea plus loin les définitions de ces deux notions qui joueront un rôle essentiel dans la suite.

Si le domaine $G$ d'intégration ne se ramène pas facilment à un pavé mais, pour fixer les idées, est contenu dans $Q^{s}$, on serait tenté de réduire l'intégrale au cas précédent en faisant $f^{*}=f$ dans $G$ ot $f^{*}=0$ partout ailleurs et en calculant $\int_{Q^{s}} f^{*}(x) d x$. Cependant, même si le domaine $G$ est bien régulier, $f^{*}$ ne sera plus, en général, à rariation bornée au sense de $H_{A R D Y}$ et KraUse, de sorte que les théorèmes sur les majorantes de la valenr absolue ne seront plus applicables. Le procédé en question, qui est souvent appliqué dans un esprit à үrai dire empirique avec la métbode de Monte Carlo, a pourtant l'avantage d'eviter le grand inconvénient des méthodes classiques d'intégration numérique qui réside dans le fait que, pour une précision donnée du résultat, la quantité de caleuls croit, grosso modo, en raison géométrique avec le nombre de dimensions. Pour obtenir une majorante de la valeur absolue de l'erreur d'intégration dans le cas envisagé, nous nous servirons d'ane généralisation, déjà considérée par HLawka [5], de la notion de discré. pance; on propose de lui donner le nom de discrépance isotrope. La majorante dont il s'agit s'exprimera alors en fonction de certains paramètres de l'inté. grande et de la discrépance isotrope d' ane façon qui généralisera un théorème de Hrawka [3]. Nous obtiendrons ensuite, pour tous les ensembles $X$ de $N$ point de $Q^{s}$, une minorante absolue de leur discrépance isotrope en fonction de $N$ et de $s$.

Notamrons. - Nous allons nous servir des notations de [7].

Les lettres avec des indices inférieurs allant de $d$ a $s$ désigneront les coordonnées d'un point qui sera, lui-mème, désigné par la même lettre en caractère gras. Nous dirons qu' un ensemble de $s$ suites finies, soit $\left\langle x_{j}^{(0)}, \ldots\right.$, $\left.x_{j}^{(m(j))}\right\rangle(j=1, \ldots, s)$ engendre une subdivision cartésienne de $Q^{s}$ si $0=x_{j}^{(0)}<$ $<x_{j}^{(1)}<\ldots<x_{j}^{m(j))}=1$ et qu' un ensemble de $2 s$ suites finies, soit $\left\langle x_{j}^{(0)}, \ldots\right.$, $\left.x_{j}^{(m(j))}\right\rangle$ et $\left\langle\xi_{j}^{(0)}, \ldots, \xi_{j}^{(m(j)+1)}\right\rangle(j=1, \ldots, s)$ engendre une double subdivision de $Q^{s}$ si les relations suivantes sont satisfaites:

$$
0=\xi_{j}^{(0)}=x_{j}^{(0)} \leq \xi_{j}^{(1)}<x_{j}^{(1)} \leq \xi_{j}^{(2)}<\ldots<x_{j}^{(m(j))}=\xi_{j}^{(m(j))+1)}=1 .
$$

Etant donnée une subdivision cartésienne de $Q^{*}$, les operateurs $\Delta_{j}$ et $\Delta_{j}^{*}$, agissant sur n'importe quelle fonction donnée sur la fermeture $\bar{Q}^{s}$ de $Q^{s}$, seront dófinies par

$$
\begin{gathered}
\Delta_{j} \varphi\left(x_{1}, \ldots, x_{j-1}, x_{j}^{(k)}, x_{j+1}, \ldots, x_{s}\right)= \\
=\varphi\left(x_{1}, \ldots, x_{j-1}, x_{j}^{(k+1)}, x_{j+1}, \ldots, x_{s}\right)-\varphi\left(x_{1}, \ldots, x_{j-1}, x_{j}^{(}, x_{j+1}, \ldots, x_{s}\right)
\end{gathered}
$$


et

$$
\begin{gathered}
\Delta_{j}^{*} \rho\left(x_{1}, \ldots, x_{s}\right)= \\
=\varphi\left(x_{1}, \ldots, x_{j-1}, 1, x_{j+1}, \ldots, x_{s}\right)-f\left(x_{1}, \ldots, x_{j-1}, 0, x_{j+1}, \ldots, x_{s}\right)
\end{gathered}
$$

pour $j=1, \ldots, s$. Dans le cas d'une double subdivision de $Q^{s}$, la même définition sera applicable avec $\xi$ à la place de $x$. Evidemment, des opérateurs avec des indices différents sont permutables entre eux. A titre d'abbréviation, nous écrirons $\Delta_{j(1)}, \ldots, j(k)$ an lieu de $\Delta_{j(1)} \ldots \Delta_{j(k)}$ et $\Delta_{j(1)}^{*}, \ldots, j(k)$ au lien de $\Delta_{j(1)}^{*} \ldots \Delta_{j(k)}^{*}$. Il est elair aussi que ces opérateurs sont permutables aveo les sommes par rapport à des variables sur lesquclles ils n'agissent pas.

Etant donnée une expression $\Phi(r, \ldots, r+k-1 ; r+k, \ldots, s)$ dépendant de la décomposition de l'ensemble des variables $r, \ldots, s$ en deux sous-ensembles $\langle j(r), \ldots, j(r+k-1)\rangle$ et $\langle j(r+k), \ldots, j(s)\rangle$, mais nullement de leur ordre,

$$
\underset{r, \ldots, s ; k}{\Sigma^{*}} \Phi(r, \ldots, r+k-1 ; r+k, \ldots, s)
$$

désignera la somme de toutes les expressions obtenues de $\Phi(r, \ldots, r+k-1$; $r+k, \ldots, s)$ en remplaçant à tour de rôle la décomposition en $\langle r, \ldots, r+k-1\rangle$ et $\langle r+k, \ldots, s\rangle$ par toutes les autres décompositions de l'ensemble de ces $s-r+1$ variables en un ensemble de $k$ variables et un ensemble de $s-r-k+1$ variables, chaque décomposition intervenant seulement une fois. Si $k=0$ ou $k=s-r+1$, l'un des ensembles de variables est vide et il n'y a pas de véritable décomposition de l'ensemble des variables $j(r), \ldots, j(s)$; cependant, pour éviter des exceptions gênantes, la somme $\Sigma^{*}$ sera interprétée dans des cas pareils comme un terme unique. Ces notations nous permettent d'exprimer relativement simplement le lemme suivant [7], qui nous sera nócessaire dans la suite et qui n'est rien d'autre $q \mathbf{u}^{\prime}$ une généralisation du lemme d'Aber.

Lempe 1.1. - Si $s$ est un entier quelconque, si $f(x)$ et $g(x)$ sont denx fonctions absolument arbitraires définies sur $Q^{s}$ et si les relations (11) sont satisfaites, on a

$(1.2)\left\{\begin{array}{l}\sum_{l(1)=0}^{m(1)-1} \ldots \sum_{l(s)=0}^{m(s)-1} f\left(\xi_{1}^{(l(1)+1)}, \ldots, \xi_{s}^{(l(s)+1}\right) \Delta_{1}, \ldots, s g\left(x_{1}^{(l(1))}, \ldots, x_{s}^{(l(s))}\right)= \\ =\sum_{k=0}^{s}(-1)^{k} \sum_{1, \ldots, s ; k}^{*} \Delta_{k+1, \ldots, s} \sum_{(1)=0}^{m(1)} \ldots \sum_{l(k)=0}^{m(k)} \\ g\left(x_{1}^{(l(1))}, \ldots, x_{k}^{(l(k)))}, x_{k+1}, \ldots, x_{s}\right) \Delta_{1, \ldots, k} f\left(\xi_{1}^{(l(1))}, \ldots, \xi_{k}^{(l(k)))}, x_{k+1, \ldots}, \ldots, x_{s}\right) ;\end{array}\right.$ 
le terme correspondant à $k=0$ est interprété de façon à faire disparaitre les signes $\Sigma$ relatifs aux variables $p(1), \ldots, p(k)$, ainsi que l'opérateur $\Delta_{1}, \ldots, k$; de même, quand $k=s$, on fait disparaitre l'opérateur $\Delta^{*}$.

Définimion 1.2. - Une fonction $f$ étant arbitrairement donnée sur $Q^{s}$, la borne supérieure $V^{(s)}(f)$ de

$$
\sum_{j(1)=0}^{m(1)-1} \cdots \sum_{j(s)=0}^{m(s)-1}\left|\Delta_{1}, \ldots, s f\left(x_{1}^{(j(1))}, \ldots, x_{s}^{(j(s))}\right)\right|
$$

par rapport à toutes les subdivisions cartésiennes de $Q^{s}$ est connue sous le nom de variation $\dot{a} s$ dimensions de $f$ sur $Q^{s}$ au sens de VITALI. Si $V^{(s)}(f)$ est finie, $f$ est dite à variation bornée au sens de VITali sur $Q^{s}$. Si la même fonction est à variation bornée au sens de VITALI quand elle est restreinte aux diverses faces de $Q^{s} \grave{a} 1, \ldots, s$ dimensions, $f$ est dite $\grave{a}$ variation bornée au sens de $\mathrm{H}_{\mathrm{ARDY}}$ et $\mathrm{K}_{\mathrm{RAUSE}}$.

Définimion 1.3. - Soit $\boldsymbol{X}$ un ensemble arbitraire de $N$ points de $Q^{s}$. Si $C^{s}$ désigne l'ensemble de tous les ensembles convexes contenus dans $Q^{s}$, $\mu(\mathbf{T})$ la mesure (à $s$ dimensions) de $T$ et $\vee\left(l^{\prime}\right)$ le nombre de points de $\boldsymbol{X}$ dans $\Gamma$, nous proposons de donner à l'expression

$$
J(\boldsymbol{X})=\sup _{\Gamma \in C^{s}}\left|N^{-1} v(\Gamma)-\mu(\Gamma)\right|
$$

le nom de discrépance isotrope de $\boldsymbol{X}$. Si. dans cette définition, on remplace $C^{s}$ par l'ensemble de tous les intervalles contenus dans $Q$, on obtient la notion classique de discrépance.

Si l'on se borne à l'ensemble de tous les intervalles contenus dans $Q^{s}$ et contenant l'origine, on obtient une notion qui a des applications importantes dans l'analyse numérique et pour laquelle on a proposé [7] le nom de discrépance extrême pour la distinguer de la discrépance en moyenne quadratique, qui, elle aussi, a des applications au calcul des intégrales multiples.

Le nom de discrépance isotrope s'explique par le caractère isotrope de $J(X)$; pour rendre cette notion complètement isotrope, on pourrait remplacer $Q^{s}$ par un ensemble convexe arbitraire de mesure 1 , mais une telle généralisation n'aurait par d'application à ce qui suit.

\section{2. - Une généralisation du théorème de Hlawka.}

Proposimion 2.1. - En conservant les notations précédentes, nous supposerons que $G$ est un ensemble mesurable d'après JoRDAN et contenu dans $Q^{s}$, mais d'ailleurs arbitraire; $\boldsymbol{X}$ étant un ensemble arbitraire de $N$ points de $Q^{s}$, nous désignerons par $\gamma_{G}(\boldsymbol{x})$ le nombre de points de $\boldsymbol{X}$ dans l'intersection de 
$G$ avec l'intervalle

$I_{x}$

$$
0 \leq \xi_{i} \leq x_{i} \quad(i=1, \ldots, s)
$$

et par $\mu_{G}(x)$ la mesure de cette intersection. Posons finalement

$$
g_{G}(\boldsymbol{x})=N^{-1} \vee_{G}(\boldsymbol{x})-\mu_{G}(\boldsymbol{x})
$$

Alors, si $f(\boldsymbol{x})$ est une fonction quelconque à variation bornée sur $Q^{s}$ au sense de $\mathrm{H}_{\mathrm{ARDY}}$ et KRAUSE, on a

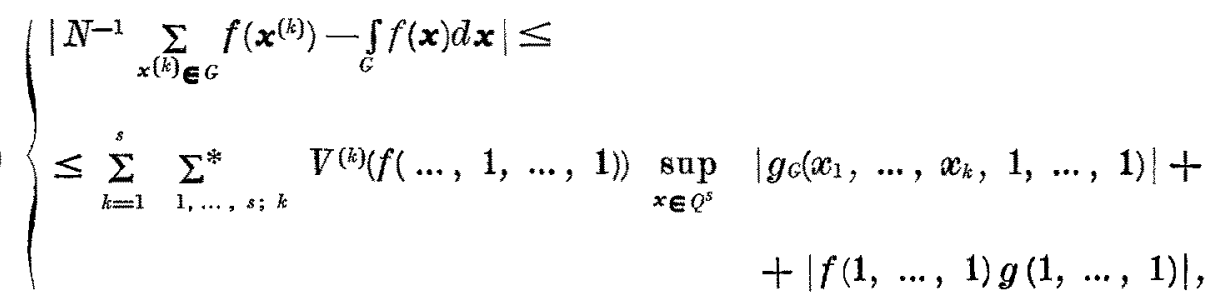

où, bien entendu, $V^{(k)}(f(\ldots, 1, \ldots, 1))$ représente la variation à $k$ dimensions au sens de Virali de la fonction obtenue de $f$ en posant $x_{k+1}=\ldots=x_{s}=1$.

Démonstration. - Comme dans la démonstration de la proposition 4 dans [7], on forme une double subdivision de $Q^{s}$ telle que chacune des coordonnées de chaque point de $\boldsymbol{X}$ soit un élément de la suite $\left\langle\xi_{j}^{(0)}, \ldots, \xi_{j}^{(w(j))}\right\rangle$ d'indice $j$ correspondant. Si. dans (1.2) avec $g$ remplacé par $g_{G}$, on passe à la limite avee

$$
\max _{0 \leq l \leq m(l)}\left(x_{j}^{(l+1)}-x_{j}^{(l)}\right) \rightarrow 0(j=1, \ldots, s),
$$

on trouve que le premier membre de cette identité tend vers

$$
\int_{Q^{s}} f(x) d g_{G}(x)=N^{-1} \sum_{x^{(k)} \in G} f\left(\boldsymbol{x}^{(k)}\right)-\int_{G} f(x) d x
$$

Quand au second membre, on remarque tout d'abord que $g_{G}(x)=0$ dès qu'au moins une coordonnée de $x$ est égale à zero, ce qui permet de remplacer $\Delta_{k+1}^{*}, \ldots, s g_{G}\left(x_{1}^{(l(1))}, \ldots, x_{k}^{(l(k))}, x_{k+1}, \ldots, x_{s}\right) \Delta_{1}, \ldots, k f\left(\xi_{1}^{(l(1))}, \ldots, \xi_{k}^{(l(k))}, x_{k+1}, \ldots, x_{s}\right)$ $\operatorname{par} g_{G}\left(x_{1}^{(l(1))}, \ldots, x_{k}^{(l(k))}, 1, \ldots, 1\right) \Delta_{1}, \ldots,{ }_{k} f\left(\xi_{1}^{(l l 1))}, \xi_{k}^{(l k))}, 1, \ldots, 1\right)$.

D'ailleurs, la somme ne diminuera pas en valeur absolue si l'on se débarasse du facteur $(-1)^{k}$, en remplaçant $g_{G}\left(x_{1}^{(l(1))}, \ldots, x_{k}^{(l k k))}, 1, \ldots, 1\right)$ par

$$
\sup _{x \in Q^{s}}\left|g_{G}\left(x_{1}, \ldots, x_{k}, 1, \ldots, 1\right)\right|
$$


et $\Delta_{1}, \ldots,{ }_{k} f\left(\xi_{k}^{(31))}, \ldots, \xi_{k}^{(l k))}, 1, \ldots, 1\right)$ par sa valeur absolue; alors la première de ces deux expressions devient un facteur de la somme par rapport à $l(1), \ldots, l(k)$, tandis $q u e$, par définition, la somme des valeurs absolues des différences finies de $f$ ne dépasse par $V^{(k)}(f(\ldots, 1, \ldots, 1))$, de sorte que l'on trouve bien (2.1).

Dans le cas particulier où $G=Q^{s}$, on retrouve la proposition 4 de [7], o.-à-d. essentiellement le théorème de Hratvra, puisque nécessairement $g_{o} s(1, \ldots, 1)=0$.

Remarquons que (2.1) donne la meilleure majorante possible dans ce sens que si, dans son second membre, on attachait à n'importe quel terme de la somme un coefficient plus petit que 1, la proposition deviendrait fausse. En effet, soit

$$
V^{(k)} f(\ldots, 1, \ldots, 1) \sup _{x \in Q^{s}}\left|g_{G}\left(x_{1}, \ldots, x_{k}, 1, \ldots, 1\right)\right|
$$

le terme dont il s' agit et soit $\left\langle x_{1}^{*}, \ldots, x_{k}^{*}, 1, \ldots, 1\right\rangle$ un point dans le voisinage dunquel $\left|g_{G}\left(x_{1}, \ldots, x_{k}, 1, \ldots, 1\right)\right|$ atteint sa borne supérieure. Si cette borne correspond à une valeur positive de $g_{G}$, posons

$$
f\left(x_{1}, \ldots, x_{s}\right)=\left\{\begin{array}{l}
1 \text { quand } x_{j} \leq x_{j}^{*}(j=1 . \ldots, k) \\
0 \text { dans tous les autres cas. }
\end{array}\right.
$$

Si au contraire cette borne correspond à une valeur négative de $g_{G}$, on remplace, dans cette définition, l'inégalité $x_{j} \leq x_{j}^{*}$ par $x_{j}<x_{j}^{*}$. Dans le premier cas, on a nécessairement $x_{j}^{*}<1(j=1, \ldots, k)$ puisque $X \subset Q^{s}$, de sorte que dans les deux cas $f(x)=0$ dès qu' une des $k$ premières coordonnées de $x$ est égale à 1.

On voit facilment que l'erreur d'intégration devient égale à sup $\left|g_{G}\left(x_{1}, \ldots, x_{k}, 1, \ldots, 1\right)\right|$. Or $V^{(k)}(f(\ldots, 1, \ldots, 1))=1$ et tous les termes $d u$ second membre de (2.1) qui ne contiennent pas cette variation s'annullent. En effet, si l'on fait une des $k$ premières coordonnées de $\boldsymbol{x}$ égale à 1 , la fonction $f$ restreinte à une telle face de $Q^{s}$ est identiquement égale à 0 , tan. dis que si au moins une des coordonnées $x_{k+1}, \ldots, x_{s}$ reste variable, la fonc. tion ne dépend pas de tous ses arguments, de sorte que la variation correspondante an sens de VITALI disparait de nouveau.

Le même raisonnement s'applique au dernier terme da second membre de (2.1). Il peut paraître étrange que la valeur de $f(1, \ldots, 1) y$ intervienne bien que le point correspondant puisse bien être en dehors du domaine d'in. tégration. Sans modifier l'erreur d'intégration et sans faire perdre à $f$ la propriété d'être à variation bornée au sens de $\mathrm{H}_{\mathrm{ARDY}}$ et Krausk, on pourrait alors modifier $f$ de façon à changer sa valeur au point $\langle 1, \ldots, 1\rangle$, mais 
il n'est pas difficile de s'appercevoir que cela modifierait les autres termes du second membre de (2.1). Si, par exemple, $s=2$ et $G$ est l'intérieur d'un cercle situé à l'intérieur de $Q^{2}$ aveo $f=1$ partout, le second nnmbre de $(2.1)$ se réduit à $\left|g_{G}(1,1)\right|$. On pourrait alors modifier la fonction $f$ en dehors du cercle en la remplaçant par une fonction $f$ dépendant de $x_{1}$ seulement de façon que $f(1,1)=0$. La majorante donnée par $(2.1)$ se réduirait alors à $\nabla^{(1)}(f(\cdot, 1))$ sup $\left|g_{G}\left(x_{1}, 1\right)\right| \geq g_{G}(1,1) \mid$, l'égalité ayant lieu seulement si $\left.V^{(1)}(f \cdot, 1)\right)=1$ (ce qui serait facile à obtenir) et si sup $\left|g_{G}\left(x_{I}, 1\right)\right|=|g(1,1)|$.

Si l'on connaît au moins des majorantes pour les variations au sens de Vrmali de $f$ et de la même fonction restreinte aux diverses faces de $Q^{\prime}$, la proposition 2.1 nous permet d'obtenir pour n'importe quel $\boldsymbol{X}$ donné des majorantes certaines pour la valeur absolne de l'erreur d'intégration, puisqu'il est toujours possible de calculer (un peu laborieusement, il est vrai) les bornes de $g_{G}$ et de la même fonction restreinte aux diverses faces de $Q^{s}$.

D'autre part, étant donné $\boldsymbol{X}$, il est désirable de connaître une majorante pour la valeur absolue de l'erreur d'integration en fonction des mêmes paramètres de l'intégrande, valable pour une classe de domaines d'intégration aussi étendue que possible. Naturellement on peat considérer différentes classes, mais bien que d'autres classes puissent présenter un certain intérêt, celle qui s'impose tout naturellemont est celle des ensembles convexes. En remarquant que si $G$ est convexe, ses intersections avec les divers intervalles $I_{x}$ intervenant dans la définition de $g_{G}$ le sont aussi, on obtient immédiatement le corollaire suivant de la Proposition 2.1.

Propostrion 2.2. - En conservant les notations précédentes, si $f$ est à variation bornés au sens de $H_{\text {ARdY }}$ et Krause dans $Q^{s}$, si $G$ est un ensemble convexe contenu dans $Q^{s}$ et si $\boldsymbol{X}$ est un ensemble arbitraire de $N$ points de $Q^{s}$, on a

$$
\left\{\begin{array}{l}
\left|N^{-1} \underset{x^{(k)} \in C}{\sum} f\left(\boldsymbol{x}^{(k)}\right)-\int f(\boldsymbol{x}) d \boldsymbol{x}\right| \leq \\
\leq J(\boldsymbol{X})\left\{\sum_{k=1}^{s} \underset{1, \ldots, s ; k}{\sum} V^{(k)}(f(\ldots, 1, \ldots, 1)+f(1, \ldots, 1)\},\right.
\end{array}\right.
$$

où $J(\boldsymbol{X})$ est la discrépance isotrope de $\boldsymbol{X}$.

Ce qui pent paraître artificiel dans cette proposition, e'est que, tandis que les conditions auxquelles $G$ et $X$ sont assujettis sont parfaitement isotropes, la condition que l'intégrande doit satisfaire dépend essentiellement des directions des axes de coordonnées. Cette dernière condition a été retenue parce qu'elle est relativement facile à vérifier; d'ailleurs, elle peut être évidemment remplacée par une condition plus forte qui est isotrope et, dans la plupart des cas, même plus facile à vérifier, à savoir celle de l'existence 
d'une dérivée partielle

continue dans $Q^{s}$.

$$
\frac{\partial^{s} f}{\partial x_{1} \ldots \partial x_{s}}
$$

3. - Une minorante absolue de la discrépance isotrope.

Par définition, la discrépance isotrope $J(\boldsymbol{X})$ de n'importe quel ensemble fini de points de $Q^{s}$ ne peut pas être plas petite que sa discrépance extrème. Hlawka [3] a démontré l'inégalité

$$
J(\boldsymbol{X}) \leq 20^{s} D(\boldsymbol{X})
$$

où $D(\boldsymbol{X})$ est la discrépance (au sens classique) de l'ensemble $\boldsymbol{X}$.

En fait, l'ordre de grandeur de $J(\boldsymbol{X})$ correspondant à une valeur donnée de $D(\boldsymbol{X})$ peut ètre proche de celui de cette majorante. Pour s'en rendre compte, on se sert du lemme suivant:

Lемme 3.1. - Si la frontière d'un sous-ensemble convexe, soit $G$, de $Q_{s}$ contient $q$ points de l'ensemble $\boldsymbol{X}$ de $N$ points de $Q^{s}$, on a

$$
J(\boldsymbol{X}) \geq q /(2 N)
$$

Démonstration. - Soit $\bar{G}$ la fermeture de $G$ par rapport à $Q^{s}$ et $\Gamma$ son intérieur. Alors $N^{-1} v(\bar{G})-\mu(\bar{G})-\left(N^{-1} v(\Gamma)-\mu(\Gamma)\right)=q / N$, ce qui entraîne soit $\left|N^{-1} v(\bar{G})-\mu(\bar{G})\right| \geq q /(2 N)$, soit $\left|N^{-1} v(\mathrm{~T})-\mu(\mathrm{C})\right| \geq q /(2 N)$.

D'autre part, pour une classe d'ensembles proposée par Huawra [4], on a

$$
D(\boldsymbol{X})=O\left(N^{-1}(\log N)^{s}\right)
$$

tandis qu'il existe, pour chacun de ces ensembles de points, une variété linéaire à $s-1$ dimensions contenant un nombre de points de cet ensemble qui est de l'ordre de $N^{1-1 / s}[6]$, de sorte que la discrépance isotrope ne peut pas être plus petite qu'un nombre de l'ordre de $N^{1 / s}$, ce qui est bien l'ordre de grandeur indiqué par (3.1) à une puissance de $\log N$ près. Ceci, naturellement, ne veut pas dire que le rapport entre les ordres de grandeur des minorantes absolues de $J$ et de $D$ pour un nombre donné de points corre. sponde toujours à (3.1), même à une puissance de $\log N$ près. Cependant, en se servant encore du lemme précédent, on trouve pour $J$ une minorante absolue d'un ordre de grandeur bien au dessus de celui de la minonante absolue de $D$; on a la proposition suivante: 
Proposition 3.2. - Pour chaque nombre $s$ de dimensions il existe une constante positive $C_{s}$ telle que pour tout ensemble $\boldsymbol{X}$ de $N$ points de $Q^{s}$ on ait

$$
J(X) \leq C_{s} N^{-(s+1) /(2 s)} .
$$

Démonstration. - On commence par quelques considérations de géométrie elémentaire. Soit $S^{s-1}$ la sphère

$$
\left\{\begin{array}{l}
x_{1}=\cos \theta_{1} \\
x_{2}=\sin \theta_{1} \cos \theta_{2} ; \\
\cdot \cdot \cdot \cdot \cdot \cdot \\
x_{s-1}=\sin \theta_{1} \ldots \sin \theta_{s-2} \cos \varphi \\
x_{s}=\sin \theta_{1} \ldots \sin \theta_{s-2} \sin \varphi
\end{array}\right.
$$

avec $0 \leq \theta_{j} \leq \pi(j=1, \ldots, s-2)$ et $0 \leq \varphi \leq 2 \pi$. Si l'on coupe $S^{s-1}$ avec une variété linéaire $\lambda$ à $s-1$ dimensions ne passant pas par l'origine, la partie de la sphère solide située du côté opposé à l'origine forme une calotte solide. La frontière de celle-ci est composée d' une sphère solide à $s-1$ dimensions située dans $\lambda$ et d' ane partie de $S^{s-1}$ formant une sphère solide dans cette variété. Si le rayon de cette dernière sphère est $\theta$, celui de la sphère dans $\lambda$ est $\sin \theta$; nous appellerons $\theta$ l'angle au centre de la calotte. Il est assez évident que pour chaque $s$ il existe une constante positive $L_{s}$ telle que pour chaque $\Theta(0<\theta<\pi / 2)$ on on puisse former, avec cet angle an centre, $2 L_{s} \theta^{1-s}$ calottes dont les intérieurs sont disjoints deux-à-deux. Comme il ne s'agit pas de la valeur exacte de $L_{s}$, on peut toujours supposer que $\Theta$ est suffisamment petit; on obtient alors une démonstration formelle de la façon suivante.

On a, sur $S^{s-1}$,

$d s^{2}=d \theta_{1}^{2}+\sin ^{2} \theta_{1} d \theta_{2}^{2}+\ldots+\sin \theta_{1}^{2} \ldots \sin ^{2} \theta_{s-3} d \theta_{s-2}^{2}+\sin ^{2} \theta_{1} \ldots \sin ^{2} \theta_{s-2} d \varphi^{2}$.

Si l'on se borne aux valeurs de $\theta_{1}, \ldots, \theta_{s-2}$ comprises entre $\pi / 4$ et $3 \pi / 4$, on a done

$$
d s^{2} \geq d \theta_{1}^{2}+2^{-1} d \theta_{2}^{2}+\ldots+2^{3-s} d \theta_{s-2}^{2}+2^{2-s} d \varphi^{2} .
$$

Posons $\varphi^{(k)}=2^{s_{i}-1} \Theta k\left(k=1, \ldots,\left[\pi /\left(2^{s-2} \theta\right)\right]\right)$ et $\theta^{(k(j))}=\pi / 4+2^{j} \Theta k(j=1$, $\left.\ldots, s-2 ; k(j)=0,1, \ldots,\left[\pi /\left(2^{+1} \theta\right)\right]\right)$ où, comme d'habitude, $[x]$ désigne le plus grand entier plus petit ou égal à $x$. En faisant $\theta_{j}=\theta_{j}^{(k(j))}, \varphi=\varphi^{(k)}$, tout en permettant à $k, k(1), \ldots, k(s-2)$ de varier indépendamment dans les limi- 
tes indiquées, on obtient, a condition que $\Theta$ soit suffisamment petit,

$$
\left(\left[\pi /\left(2^{2} \Theta\right]+1\right) \ldots\left(\left[\pi /\left(2^{s-1} \Theta\right)\right]+1\right)\left[\pi /\left(2^{s-2} \Theta\right)\right]>\pi^{s-1} \Theta^{1-s} 2^{1-s(s+1) / 2}\right.
$$

différents points de $S^{s-1}$ situés deux-à-deux à des distances égales $2 \Theta_{\text {cha }}$ au moins dans la métrique de $S^{s-1}$, de sorte que les intérieurs des calottes correspondantes avec des angles an centre égaux à $\theta$ sont disjoints deux-à-deux. Autrement dit, on peut prendre $L_{s}=\pi^{s-12^{s(s+1) / 2}}$.

Il est facile d'obtenir une minorante commode de la mesure de ces calottes. En effet, comme elles sont toutes égales, on peut supposer qu'il s'agit de celle qui est obtenue en coupant $S^{s-1}$ avec la variété linéaire $x_{1}=\cos \Theta$. En désignant par $x^{(s-1)}$ la mesure de la sphère solide à $s-1$ dimensions de rayon 1 , on trouve que la mesure de la calotte est égale à

$$
x^{(s-1)} \int_{\theta}^{0} \sin ^{s-1} d(\cos \theta)=x^{(s-1)} \int_{0}^{\Theta} \sin ^{s} \theta d \theta=(2 s+2)^{-1} x^{(s-1)} \theta^{s+1}
$$

dès que $\Theta$ est suffisammenl petit.

Cela posé, la démonstration de la proposition devient très simple. Nous inscrivons dans $Q^{s}$ une sphère de rayon $\frac{1}{2}$. Le plus grand nombre de calottes avec des angles aux centres égaux à $\Theta$ n'ayant pas, deux-à-deux, de points intérieurs en commun reste le mème que précédemment, tandis que leur mesure est divisée par $2^{s}$. Etant donné un ensemble $\boldsymbol{X}$ de $N$ points de $Q^{s}$ ayant une discrépance isotrope $J\left(\boldsymbol{X}_{)}\right.$, choissons $\theta$ de façon que

c'est à dire

$$
J(\boldsymbol{X})=2^{-s}(2 s+2)^{-1} \gamma_{(s-1)} \Theta^{s+1},
$$

$$
\ominus=\gamma_{s} J(\boldsymbol{X})^{1 /(s+1)}
$$

où $\left.\gamma_{s}=2(s+1) / x^{(s-1)}\right)^{1 /(s+1)}$. Comme on peut toujours supposer que $J(\boldsymbol{X})$ est petit, ce qui rend $\Theta$ petit, il est légitime de ce servir des formules établies précédemment. On forme alors, avec $\Theta$ pour angle au centre, $2 L_{s} \Theta^{1-s}$ calottes dont les intérieurs sont disjoints deux-à-deux. Leur mesure étant plus grande que $J(X)$, chacune d'elles contient dans son intérieur au moins un point de $\boldsymbol{X}$. Ayant choisi dans chaque calotte un de ces points, on forme leur enveloppe convexe. Il est clair que la frontière de celle-ci contient tous les points choisis. Par suite, en vertu de (3.5) et d'après le Lemme 3.1, on a

$$
J(\boldsymbol{X}) \geq L_{s} \gamma_{s}^{1-s} J(\boldsymbol{X})^{(1-s))(1+s)} N^{-1}
$$

d'où l'on déduit (3.4) aree $\left.C_{s}=\left(L_{s} \gamma_{s}^{1-s}\right)^{(s-1) /(}\right)$. 


\section{4. - Conclusion.}

Il reste à résoudre deux questions difficiles, à savoir: (1) Est-ce qu'il existe, dans plus d'une dimension, des ensembles de points ayant des discrépances isotropes d'un ordre de grandenr correspondant à la Proposition 3.2? (2) Comment pout-on former systématiquement des ensembles ayant des discrépances isotropes satisfaisantes au point de vue des applications à l'analyse numérique?. On espère commencer à trouver des réponses partielles à à ces questions en étudiant la discrépance isotrope des ensembles aléatoires. Bien entendu, celle-ci sera presque toujours d'un ordre de grandeur plus grand que celui de la minorante qui vient d'être trouvée, mais on pent s'attendre à ce que cette discrépance soit beaucoup plus petite que celle de la plupart des ensembles de points construits jusqu'à present en vue d'obtenir une discrépance extreme $D(X)$ petite. Il vaut cependant la peine de remarquer que l'on ne sait rien au sujet de la discrépance isotrope d'une classe d'ensembles proposée par J.H. HaLton [1].

O'est seulement la connaissance d'ensembles de points à différents nombres de dimensions ayant des discrépances isotropes satisfaisantes qui permettra de pratiquer sur une base rationnelle le calcul d'intégrales multiples sur des domaines convexes arbitraires dans un nombre fini de dimensions par la méthode de Morte $\mathrm{O}_{\Delta \mathrm{RLO}}$, on plutôt par une méthode qu'on a proposé [8] d'appeler quasi-Monte-CARLO.

\section{LITERATURE}

[1] J.H. HALTON, On the efficiency of certain quasi-random sequences of points in evaluating multidimensional integrals. Numer. Math. 2 (1960), pp. 84.90 et. 196.

[2] J.H. HALTON et S.K. ZAREMBA, The extreme and $L^{2}$ discrepancies of some plane sets. Monatsh Math. 73 (1969), pp. 316-328.

[3] E. HLAWKA, Funktionen von beschränkter Variation in der Theorie des Gleichverteilung, Ann. Mat. Pura Appl. (IV) 64 (1961), pp. 325-334.

[4] - -, Zur angenäherten Berechnung mehrfacher Integrale, Monatsh. Math. 66 (1962), pp. 140-151.

[5] - -, Diserepancy and uniform distribution of sequences, Comp. Math. 16 (1964), 88.91.

[6] S. K. ZAREMBA, Good lattice points in the sense of Hlawka and Monte Carlo integration, Monatsh Math. 72 (1968) pp. 264.269.

[7] - - Some applications of multidimensional integration by parts, Ann. Polon. Math. 21 (1968), pp- 85.96.

[8] - -, The mathematical basis of Monte Carlo and quasi-Monte Carlo methods, SIAM Rev. 10 (1968), pp. 303*314; reproduit dans SIAM Studies in Applied Mathematies III. 